\title{
Government Data Sharing based on Blockchain
}

\author{
Junfang Zeng \\ Institute of Automation, Chinese Academy of Sciences \\ junfang.zeng@ia.ac.cn
}

\begin{abstract}
With the development of smart city, the cooperation between government sectors is becoming more and more frequent, breaking the information island and big data sharing has become an inevitable trend. The traditional way of government data sharing is mainly based on one unified data center, which has high construction cost and maintenance cost, and fails to fully consider the sectoral interests, authority, security and traceability, so there are still many barriers in government data sharing. Taking advantage of the features of blockchain technology, such as distributed ledger, smart contract, data trust and traceability, this paper proposes an application model of government data sharing based on blockchain, which uses a unified data sharing model and autonomous authority management to provide efficient and safe bus service for distributed government big data sharing.
\end{abstract}

\section{CCS CONCEPTS}

- Blockchain; • Big Data sharing; • Distributed;

\section{KEYWORDS}

Data sharing model, Authority management, Bus service, City brain

\section{ACM Reference Format:}

Junfang Zeng and Yu Liu. 2021. Government Data Sharing based on Blockchain. In 2021 The 3rd International Conference on Blockchain Technology (ICBCT '21), March 26-28, 2021, Shanghai, China. ACM, New York, NY, USA, 6 pages. https://doi.org/10.1145/3460537.3460562

\section{INTRODUCTION}

With the development of smart city, the cooperation between government sectors is becoming more and more frequent, which is accompanied by a large number of data exchange, breaking the information island and big data sharing has become an inevitable trend. Government data sharing needs to better protect data security, prevent information barriers caused by sectoral conflicts of interests, promote the aggregation and effective use of government data, and the goal is to establish a data-driven "city brain" to achieve scientific and efficient city governance and public services.

At present, the mainstream way of government data sharing is to build one unified big data center, which has disadvantages in five aspects: 1) The sharing cost is high, due to the large scale of data,

Permission to make digital or hard copies of all or part of this work for personal or classroom use is granted without fee provided that copies are not made or distributed for profit or commercial advantage and that copies bear this notice and the full citation on the first page. Copyrights for components of this work owned by others than ACM must be honored. Abstracting with credit is permitted. To copy otherwise, or republish, to post on servers or to redistribute to lists, requires prior specific permission and/or a fee. Request permissions from permissions@acm.org.

ICBCT '21, March 26-28, 2021, Shanghai, China

(C) 2021 Association for Computing Machinery.

ACM ISBN 978-1-4503-8962-4/21/03 ..\$15.00

https://doi.org/10.1145/3460537.3460562

\author{
Yu Liu \\ Institute of Automation, Chinese Academy of Sciences \\ yu.liu@ia.ac.cn
}

frequent data exchange takes much time cost, and the construction and maintenance cost of building a big data center is high; 2) The data security is hard to guarantee, the huge amount of data in the center brings great difficulties for fine grained data authority and security; 3) The interests of sectors are easy to be infringed, the data aggregation leads to the fuzzy boundary of sectoral data, resulting in unclear rights and responsibilities; 4) Privacy protection is difficult to guarantee, the government big data involves a large number of sector's secrets and citizens' privacy, which may bring adverse consequences if it is not properly disclosed; 5) The efficiency of decision-making is not high, as the data is collected by the bottom layer, then reported, audited and processed layer by layer, which will affect the decision-making efficiency in case of emergency.

Blockchain technology $[1,4]$ stores data in the form of blocks connected in chronological order, and realizes data encryption and sharing through hash operation, timestamp, asymmetric encryption technology, so as to form a chain that cannot be tampered with. Taking advantages of the features of blockchain technology, such as distributed ledger, smart contract, data trust and traceability [3, 67], this paper proposes an application model of government big data sharing based on blockchain, which mainly realizes the data sharing between government sectors from two aspects: unified data sharing model and autonomous authority management. Compared with the traditional centralized mode, it can better protect sectoral interests while ensure data security, providing a feasible solution to the city big data sharing.

\section{FRAMEWORK OF BLOCKCHAIN BASED DATA SHARING}

Using the blockchain based bus structure to realize the government data sharing, as shown in Figure 1. The core idea is that each sector builds or transforms the local database, "city brain" establishes the blockchain distributed ledger of the resource directory based on a unified data sharing model (including business data model, metadata model, directory data model, trading data model and authority data model), and manages it through autonomous authority management (including user management, access control and authorization management), so as to form a "logically unified" and "physically dispersed" decentralized structure, and provide bus services (directory service, trading service, authority management etc.) for government data sharing.

The framework of government data sharing platform is built under the bus structure based on blockchain. Here, "logical unified " is that all government sectors first build or transform the database based on the unified business data model, then upload the resource directory according to the unified standard, and then exchange and share the data through the unified access interface. Each sector is no longer isolated, but linked together through a unified data sharing model, forming a logically unified whole; while "physically dispersed" is that the business data of each sector is only stored 


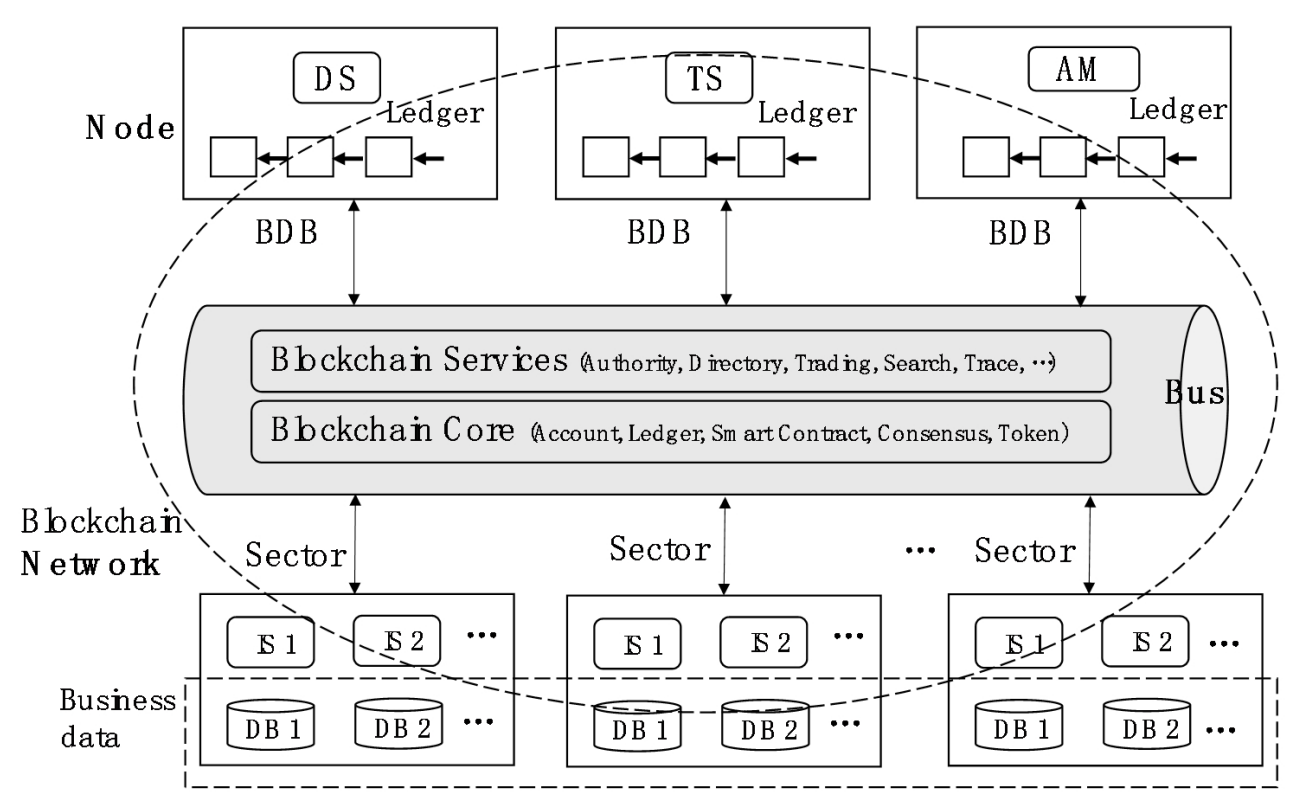

B: In form ation System ;D S:D irectory Service;TS:Trading Service;AM :A uthority M anagem ent Sector:Govemm ent sector, platfom user;BD B:Big Data Bureau, p htfom m anager

Figure 1: Blockchain based government data sharing decentralized structure.

in the local database, and the sector can autonomously manage its users, authorization and data access. Because the production and storage of business data are still in sector nodes, the burden of communication, computing and storage of government data sharing is reduced compared with the central mode. The trading data of e-Gov data, traffic data, energy and power data, medical data, industrial data and other fields are stored on blockchain, automatically form a decentralized ledger which manages the ledger data across multiple nodes. Therefore, the data sharing system based on blockchain network is more reliable.

The framework of blockchain based big data platform of city brain is shown in Figure 2, with five layers from bottom to top: data layer, blockchain core layer, blockchain service layer, decentralized application layer and user interface layer [9-10]. Based on the underlying technology of blockchain, the smart contracts [5] and incentive mechanism are customized based on business logic [8], and the decentralized application DAPPs in various business fields are developed, covering the whole business process of city brain big data platform.

- 1. Data layer: provides business database and blockchains storing metadata, authority data, directory info, trading data, tracing data etc. Multi signature permission control is used to ensure data security.

- 2. Blockchain core layer: based on the underlying technology of alliance blockchain [14], it provides account, node management, decentralized ledger, smart contracts, consensus algorithms [2], token mechanism, authorization, synchronization, data privacy, search and data trading etc.
- 3. Blockchain service layer: provides bus services of account registry, identity authentication, authority management, data directory, data trading, data query and tracing etc., and APIs to support upper application development.

- 4. Decentralized application layer: based on the data collected by IOT devices [13] and information systems, the DAPPs of government sectors run on the business blockchains, covering e-government DAPP, transportation DAPP, environment DAPP, energy DAPP etc.

- 5. User interface layer: presents the results of data analytics in a visual way to support decision-making [15-16]. Users include government sectors, Big Data Bureau and other users (enterprises, institutions, big data development teams, the public and so on). Web version of B/S structure and mobile APP version are provided.

\section{APPLICATION MODEL OF BLOCKCHAIN BASED DATA SHARING}

Participants of the traditional centralized big data platform, for the sake of sector interests and data privacy, have low enthusiasm for sharing data. The consortium blockchain [17] based data sharing platform has the features of full participation, autonomous authority control, data traceability, sharing incentive, etc., which provides a new technical approach for solving the problems in government data sharing.

\subsection{Data sharing model}

Due to the different database systems, data formats, data coding and access interfaces adopted by different government sectors and 


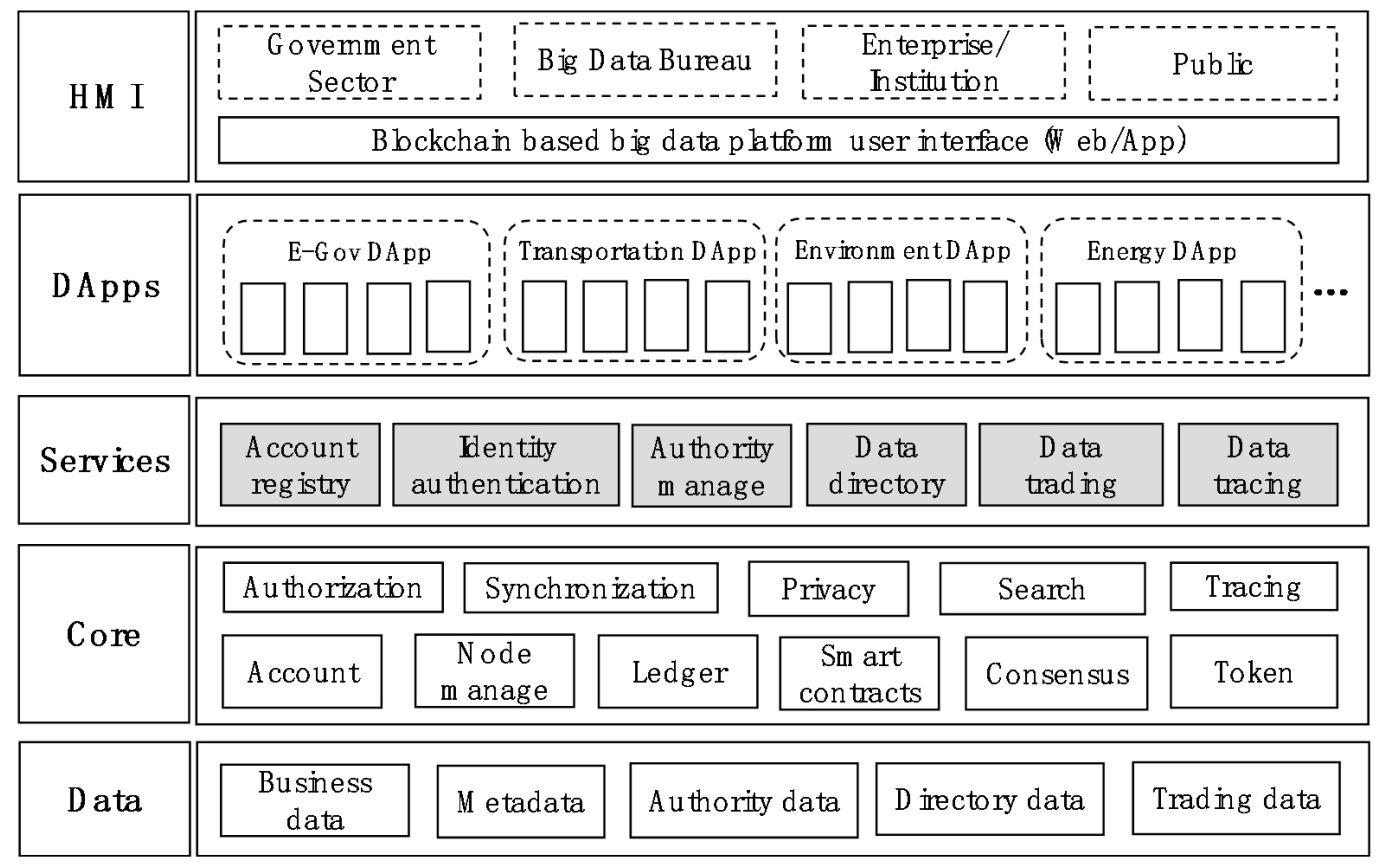

Figure 2: Framework of blockchain based government data sharing platform.

the lack of support by a unified data model, the data formats and metadata standards are inconsistent, the processing technologies and application platforms adopted are different, and the data interfaces are not interlinked, so it is difficult to share data because of the diversity [18]. Therefore, the construction of a unified data sharing model is the premise of data sharing.

According to the practical business requirements and referring to the existing relevant standards, the model system formulated by the "city brain" project office through consultation with various sectors, consists of five parts: business data model, metadata model, directory data model, trading data model and authority data model, as shown in Figure 3. For the business data, the definition of database table fields and the format of unstructured data are specified; for the access interface, it is specified that all sectors adopt the Web Services interface uniformly; for the resource directory, all sectors upload directory info to the directory manager for cataloging business data according to the specified data producer, timestamp, data format, content, access path, server IP etc.

1) Business data model

Different government sectors have different data codes and formats. In order to realize data sharing, business data should be standardized. A unified business data model meeting the sharing needs of government sectors should be built. Government data includes structured data and unstructured data. For the structured data (DB dataset), specify the name, type, length, content and description of table fields. For the unstructured data (file, image, video, etc.), specify the format, coding, resolution, frames per second, etc. Then based on the established unified business data model, the sector database is constructed or transformed.

2) Metadata model
In order to facilitate users to quickly understand the data, we need to build metadata (the data about data). Under the blockchain based bus structure, all sectors need to reach consensus on what to be shared, resource content and data organization, so a unified metadata model need to be established. Metadata standards include the representation, structure and format of metadata content, the specific description of metadata terms and data dictionary. It mainly includes the name, content overview, producer, storage location, media type and resource backup of data resources; the format structure and description of metadata content; the specific description methods of metadata terms, to define each element and modifier, etc.

3) Directory data model

In order to facilitate the data access of users, the platform provides a convenient navigation directory, namely resource directory. Resource directory is used to show the overall structure and relationship of data resources, and guide users to find and use data resources quickly and effectively. Resource directory provides directory information of government big data resources. To realize data sharing, each sector needs to provide its own resource directory according to the unified standard, publish it to the blockchain network through the smart contracts, and then organize it by the platform according to data content, access path, data catalog, etc., and save the directory information to the blockchain (directory data ledger), each block is linked to each other according to the generated time sequence. The directory service based on blockchain has the features of multi center [11-12], non-tamperable, traceable access, and traceable flow, which is more granular, safe and reliable compared with the traditional directory service mode.

4) Trading data model 


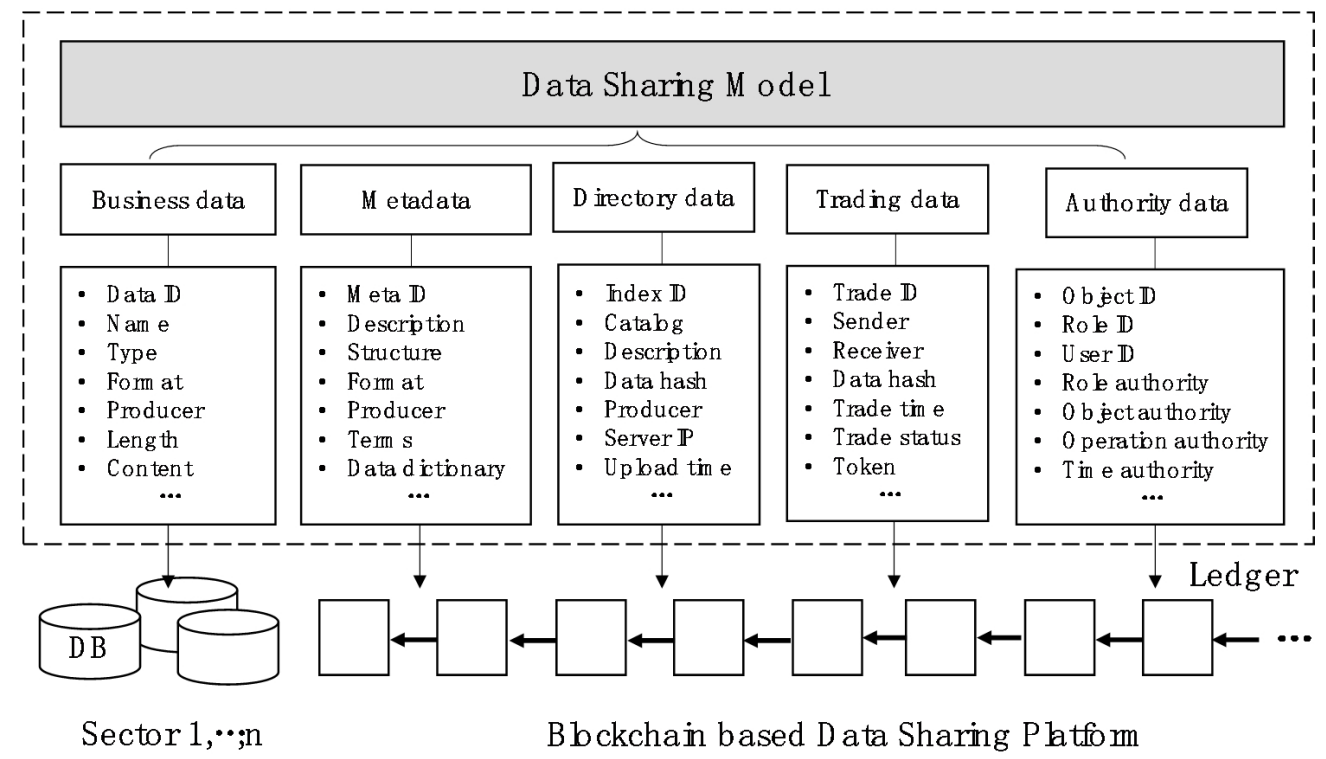

Figure 3: Data model of blockchain based government data sharing.

To realize the interconnection of different sectors, it needs to be based on a unified trading data model and access interface. The trading data provides directory information such as exchange service address. The trading data model is defined as " $4 \mathrm{Wh}$ " $<\mathrm{Who}$, What, Where, When, How $>$, Who: sender, receiver; What: data to be shared; Where: server IP to store the data; When: trade time; How: trade status and token needed. The blockchain data sharing platform also needs to provide a unified access interface, equivalent to a data interaction protocol (API, SDK, Web Services), which specifies the format of trading data and the way of data processing. For example, the Web Services interface uses standard XML files to describe all the details of data interaction, including message format, transmission protocol and location.

5) Authority data model

Authority data mainly includes role authority, operation authority, object authority and time authority. Therefore, a four tuple can be formed as $<$ Role, Operation object, Operation permission, Effective period $>$ to realize the permission configuration of role.

The business data of each government sector is stored locally in its own servers, while the metadata, authority data, directory data and trading data are stored in blockchains on the data sharing platform.

\subsection{Autonomous authority management}

Government data involves sector's secrets and public privacy, so strict authority management is needed. In the traditional sharing process based on big data center, the power and responsibility of each sector is fuzzy, and the data security is hard to fully guarantee. In the blockchain based mode, each sector can specify the access rights of other sectors by itself through autonomous authorization management, which can avoid the problem of unclear rights and responsibilities.
Each sector manages its database autonomously through smart contract mechanism, including user management, access control and authorization management. Government sectors edit smart contracts (write Scripts) according to data sharing requirements, specify data access rights, then publish contracts to their smart contract accounts, and the smart contracts are validated through the blockchain consensus mechanism. By using account registry, identity authentication and authorization management of blockchain, the orderly flow of information, authorization sharing and information interaction process between nodes in the cooperative operation network can be recorded, traceable and non-tamperable.

1) User management

The process of government data sharing involves multiple users, and effective user management is the premise of authority management. User management manages participants involved in the big data system and the relationship between them, mainly including user Add, Delete, Modify and Query, as well as View the definition of the relationship between users.

2) Access control

Role based access control (RBAC) converts the rights of an operation object into the rights of a role, which is fine grained and flexible, greatly reduces the complexity of the system, and reflects the organizational structure of the system. By configuring different roles to a certain user, different rights are assigned to users.

3) Authorization management

The main purpose of user rights management is to ensure the security of the system. To realize authorization management, firstly define the operation object and operation permission in the system, and then set the operation permission of role to operation object. 


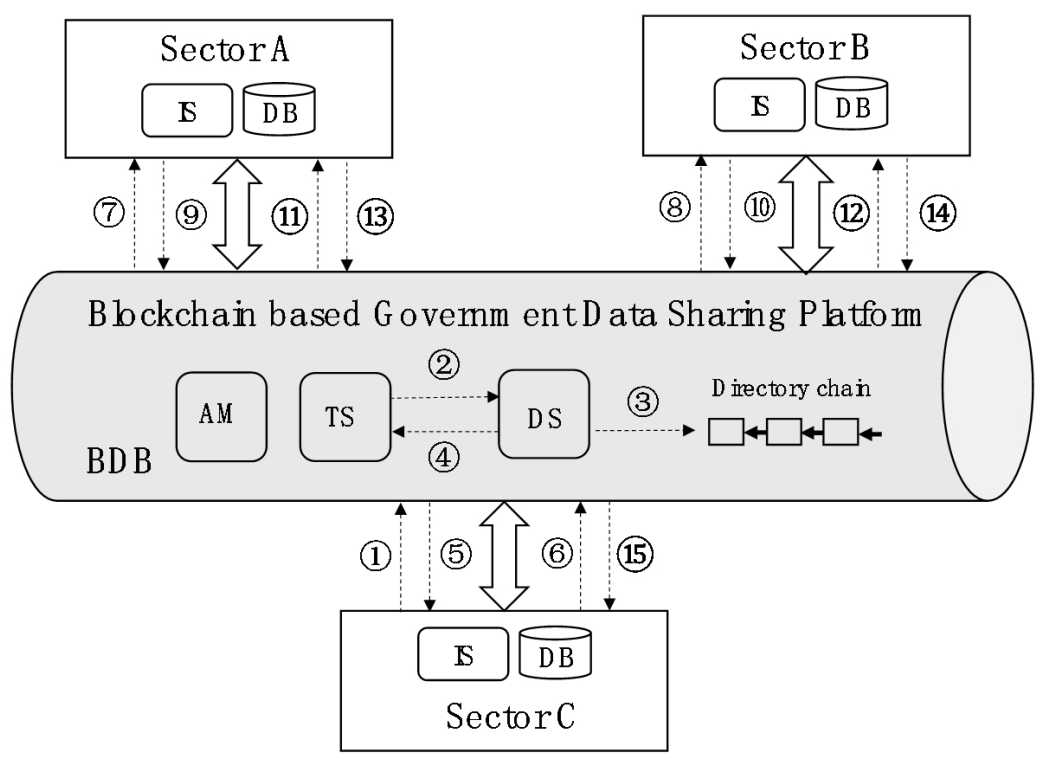

S : Inform ation System ;D S:D irectory Service; TS:Trading Service;AM :Authority M anagem ent Sector:Govemm en tsector, $\mathrm{phtform}$ user;BDB:Big Data Bureau, $\mathrm{p}$ htform m anager

Figure 4: Workflow of blockchain based government data sharing.

\subsection{Application mode of government data sharing}

The application mode of government data sharing based on blockchain is described as follows:

1) Based on the unified data sharing model, each sector builds or transforms its own database, stores business data in local servers according to the unified standard, and submits metadata and directory data to the Directory Service system for cataloging according to the unified standard, so as to realize the interconnection of sectors through the unified access interface.

2) Each sector has its own authority management. During data sharing, each sector autonomously carries out authority management by publishing and executing smart contracts, including user management, role based access control and authorization management.

3) All sectors share data through blockchain bus structure. In data sharing, users first access the directory service DAPP, query the server address from the resource directory chain, obtain the storage location of data resources, and then access the server where the business data is located for data trading.

Relevant personnel of government sector need to register a legal account of the blockchain and generate their own public key and private key. When data sharing activities occur, each block needs the public key and private key of both parties to run. The process of data interaction (data trading) on the chain: 1) user A and user $B$ have data interaction; 2) trading data between users is stored in the block; 3) user B broadcast the block to all nodes of the whole network; 4) each node is signed by A and B after verifying that the interaction is correct; 5) the block is subsequently added to the blockchain, and the trading record cannot be modified and deleted;
6) the trading data is put on the chain. Then the data trading process has completed.

Data trading of big data sharing platform is automatically executed through the smart contract mechanism of blockchain. Smart contracts are coded and published to blockchain, executed under specific circumstances. The workflow of data sharing is as shown in Figure 4

For instance, Sector $\mathrm{C}$ needs to query information $\mathrm{X}$ and information $Y$ to handle its business. The interaction process with Sector $\mathrm{A}$ and Sector $\mathrm{B}$ is done as follows:

(1) Sector $\mathrm{C}$ user submits info $\mathrm{X}$ and info $\mathrm{Y}$ access requests to the Trading Service (TS) of the data sharing platform.

(2) TS sends info X and info Y query requests to the Directory Service (DS) of the platform.

(3) DS first queries whether there is info $\mathrm{X}$ and info $\mathrm{Y}$ from the resource directory chain. If there is, returns the storage location (server IP) of info X and info Y; otherwise, returns a Null value.

(4) DS returns the location information ("info $X$ in Sector A, info $\mathrm{Y}$ in Sector B") to the TS.

(5) TS returns "info X in Sector A, info Y in Sector B" to Sector C.

(6) Sector $C$ user submits to TS the info $X$ request for accessing Sector A and the info Y request for accessing Sector B, and together with the user ID.

(7) (8) TS sends the access requests of info $\mathrm{X}$ and info $\mathrm{Y}$ to Sector $A$ and Sector B respectively.

(9) 10 Sector A and Sector B submits the user authority query request to the Authority Management (AM) of the platform according to the user ID. 
(11) 12 AM respectively returns the authority info "the user can Read Only the info X in Tstart-Tend" to Sector A and "the user can Read Only the info Y in Tstart-Tend" to Sector B.

(13) 14 Sector A and Sector B respectively packages "info X and its metadata" and "info Y and its metadata", then returns them to TS via Web Services interface.

(15) TS returns the data packets obtained from each system to Sector C via Web Services interface, meanwhile stores trading data on the blockchain.

\section{CONCLUSION}

Aiming at the challenge of data openness and data security in smart city data sharing, this paper proposes an application model of government data sharing based on blockchain, the unique advantages of blockchain technology provide a new technical solution. Its core idea lies on a unified data sharing model and autonomous permission management, which provides resource directory service, data trading service and permission management service. Compared with the traditional sharing mode of one Big Data Center (BDC), this model not only realizes the interconnection of government sectors with less cost, but also ensures the interests of sectors, and data security through autonomous authority management, and trading service records the data interaction on the blockchain, which is traceable and non-tamperable, greatly reduces the difficulty of data synchronization and maintenance. The blockchain based propose is applicable to the development of government data sharing and artificial intelligence application in smart city.

Government data is a valuable resource for a city and the key to data-driven city governance and intelligent decision-making. Further introduce the token mechanism of blockchain, establish a market-oriented data sharing system [19], encourage government sectors to share data, obtain revenue incentives, such as government performance evaluation and city public services, to form an open and autonomous ecosystem of government data co-producing and sharing, and provide big data support for city brain construction and data value deep mining using artificial intelligence technology.

Blockchain technology is becoming the trend of digital economy in the future. The blockchain based city big data platform provides the development opportunity of sharing and co-governance for social governance. Blockchain can not only provide high quality and effectiveness of data opening from technical level, but also bring new city management mode and new production relations. Through the government's open access interface, data information can be provided to other relevant users for free or with appropriate charges. Data users could process the acquired data to achieve new value-added application, and further provide data service to other users. This process is approaching the goal of forming data sharing open ecology countrywide and even worldwide.

\section{ACKNOWLEDGMENTS}

This research is supported by National Defense Basic Scientific Research Program (Grant no: JCKY2019203C029).

\section{REFERENCES}

[1] Atul Adya, Paramvir Bahl, Jitendra Padhye, Alec Wolman, and Lidong Zhou. 2004. A multi-radio unification protocol for IEEE 802.11 wireless networks. In Proceedings of the IEEE 1st International Conference on Broadnets Networks (BroadNets'04) . IEEE, Los Alamitos, CA, 210-217. https://doi.org/10.1109/BROADNETS. 2004.8

[2] DWYER G P. The economics of bitcoin and similar private digital currencies[J]. Journal of Financial Stability, 2015, 17: 81-91.

[3] BENTOVI, LEEC, MIZRAHIA, et al. Proof of Activity: Extending Bitcoin's Proof of Work via Proof of Stake[J]. Acm Sigmetrics Performance Evaluation Review, 2014, 42(3): 34-37.

[4] LYU Shining. YAN Yong, DING Qi, et a1. Application of blockchain in energy internet: advantage, scenario and case[J]. Zhejiang Electric Power, 2017, 36(3): $1-4$.

[5] YUAN Yong, WANG Feiyue. Blockchain: the state of the art and future trends[J]. Acta Automatica Sinica, 2016, 42(4): 481-494.

[6] Christidis, K. and Devetsikiotis, M. Blockchains and smart contracts for the Internet of Things [EB/OL]. [2016-05-10]. IEEE Access Vol.4: 2292-2303.

[7] https://doi.org/10.1109/ACCESS.2016.2566339.

[8] YAN Yong, ZHAO Junhua, WEN Fushuan, et a1. Block-chain in energy systems: concept, application and prospect[J]. Electric Power Construction, 2017, 38(2): $12-20$.

[9] YANG Dechang, ZHAO Xiaoyu, XU ZiXiao, et al. Developing status and prospect analysis of blockchain in energy internet[J]. Proceedings of the CSEE, 2017, 37(13): 3664-3671.

[10] Dept. of Environment \& Resources Conservation NDRC, National Energy Conservation Center. Technical specifications for online monitoring of key energy consumption units (Trial), the first publishing of 12 specifications [EB/OL]. [201805-11].

[11] http://www.chinanecc.cn/website/News!view.shtml?id=207786.

[12] Glaser, F. Pervasive decentralisation of digital infrastructures: a framework for blockchain enabled system and use case analysis. 50th Hawaii International Conference on System Sciences (HICSS 2017), Waikoloa, Hawaii, USA, 1-14.

[13] Chen S, Shi R, Ren Z, et al. A blockchain based supply chain quality management framework[C]. 14th IEEE Inter-national Conference on E-Business Engineering (ICEBE 2017), Shanghai, China, 2017:172-176.

[14] YANG Mingtong, ZHOU Buxiang, DONG Shen, et al. De-sign and dispatch optimization of microgrid electricity market supported by blockchain [J]. Electric Power Automation Equipment, 2019, 39(12): 155-161.

[15] MICHAEL C. Ethereum used for 'first' paid energy trade using blockchain technology [EB/OL]. [2016-04-11]. http://www.coind-esk.com/ethereum-used-firstpaid-energy-trade-using-blockchain-technology/.

[16] IRINA S W. Block Charge: the blockchain based solution for charging electric cars [EB/OL]. [2016-10-27]. https: //www. 1inkedin. corn/pulse/blockchargeblockchain based-solution charging-ears/.

[17] Buterin, V. On public and private blockchains. Ethereum Blog, Crypto Renaissance Salon. 7th August 2015.

[18] YANG Ting, ZHAO Junjie, ZHANG Weixin, et al. Data blockchain generation algorithm of cyber physical power system[J]. Electric Power Automation Equipment, 2018, 38(10): 80-86.

[19] MA Tiannan, PENG Lilin, DU Ying, et al. Competition game model for local multimicrogrid market based on blockchain technology and its solution algorithm [J]. Electric Power Automation Equipment, 2018, 38 (5): 198-210. 